\title{
Caring for Patients' Families (or Lack of Family) in Neurocritical Care
}

\author{
David Y. Hwang ${ }^{1}$
}

Published online: 15 September 2017

(C) Springer Science+Business Media, LLC 2017

This issue of Neurocritical Care presents several articles focused on the families and surrogate decision makers of critically ill patients with acute neurologic injury (ANI) and collectively highlights several key points. First, as the general critical care community has recognized for their patients' families [1], families of patients admitted to neuroscience intensive care units (neuro-ICUs) are not just put in tough, unfamiliar situations, but are at real risk for adverse psychological outcomes. Second, while developing interventions to improve family outcomes is crucial [2], implementing new practices and proving that they improve family experiences are not necessarily easy tasks, even when a practice seems as though it should be a "slamdunk." Third, goals-of-care decision-making is an area where improvements could be impactful, yet clinicians and families can be far apart on issues even as granular as the exact words used to express prognosis. Finally, as challenging as issues for families are, the management of incapacitated patients who lack surrogate decision makers can be even tougher. While caring for families is often (and rightfully) thought of as part of the "art" of neurocritical care, the articles in this issue demonstrate the value of scientific study in understanding best family-centered practices.

For years now, the world of oncology has incorporated the psychosocial care of patients and families into routine treatment. To draw attention to the psychosocial needs

David Y. Hwang

david.hwang@yale.edu

1 Division of Neurocritical Care and Emergency Neurology, Department of Neurology, Yale School of Medicine, P.O. Box 208018, New Haven, CT 06520, USA among neuro-ICU patients and their caregivers, Vranceanu and colleagues [3] conducted a single-center survey study comparing anxiety and depression symptoms among 81 ANI patients hospitalized in the neuro-ICU and their 95 caregivers, to 350 outpatients who had recently received a diagnosis of incurable cancer and their 275 caregivers as well. The authors were careful to use the well-validated Hospital Anxiety and Depression Scale (HADS) for assessing outcomes. Controlling for demographic differences between cohorts, the study team found not only that patients with ANI reported greater anxiety symptoms than those with cancer, but also that ANI caregivers had worse symptoms of depression than caregivers for cancer patients.

Several aspects of these comparisons are worth emphasizing. First, the ANI patients and caregivers were recruited in the hospital within 2 weeks of neuro-ICU admission, while the cancer patients and caregivers were recruited in clinic or by telephone. It is not difficult to imagine that being in an inpatient environment alone may have contributed to higher rates of anxiety among patients and depression among caregivers. Second, while differences in psychological outcomes among cohorts were found, the absolute HADS scores for each of the cohorts were not necessarily high enough to conclude definitively that the average patient or caregiver in either the ANI or cancer group had clinically significant anxiety and/or depression [4]. However, setting aside the debate over what clinically significant "cutoffs" are for anxiety and depression HADS scores, one can still conclude that the differences between disease groups justify an assertion that the psychosocial care for neuro-ICU patients and their caregivers should at least approach the level that is routine for patients diagnosed with cancer. Assuming one accepts this assertion, the field of neurocritical care certainly has opportunity for improvement. 
The formal testing of practice changes designed to meet the needs of patients' caregivers in the neuro-ICU may, on the surface, feel somewhat unnecessary to some. Many examples of such practice changes-allowing families to observe or participate in work rounds, designing protocols for family meetings - may seem as though they are "common sense" and low risk. However, definitively proving the efficacy of a family-centered practice change can be difficult to do. Creutzfeldt et al. [5] conducted a single-center study of the effect of a daily palliative care needs checklist on family satisfaction with ICU care and family ratings of depression and post-traumatic stress disorder (PTSD) following discharge. The study team leveraged their existing two-team system and implemented the daily checklist on morning work rounds for only one of their teams over a 7-month study period, allowing for a comparison of family outcomes between teams. The checklist itself screened for three key aspects of clinical care applicable to majority of neuro-ICU patients - pain control, adequate social support, and appropriate goals-of-care. Such an intervention might seem like low-hanging fruit for improving family experiences and psychological outcomes.

Yet, despite enrolling nearly 200 patients and their family members into the study, the team was mostly unable to demonstrate a difference between groups with respect to family satisfaction (on various items taken from the validated FS-ICU survey) and family ratings of depression or PTSD, several months after hospital admission. In fact, a DSM-IV-based algorithm the authors used for determining PTSD suggested worse scores for the checklist-screening cohort versus the control cohort. While these findings must be taken with a grain of salt given the multiple comparisons made in this single-center study, they are nevertheless notable in the context of a recent high-profile study questioning whether palliative care consultation may be inadvertently associated with increased PTSD among families [6]. At the very least, there is a clear message to not take for granted that any intervention that makes theoretical sense will necessarily be proven efficacious. Neurocritical care would greatly benefit from the continued aggressive pursuit of interventions that can be rigorously proven to improve family outcomes.

Building upon one of the aspects of care in the checklist above, discussions of goals-of-care and thus prognosis run a particularly high risk for misunderstanding between patients' families and clinicians. While the world of general critical care has increasingly paid attention to discordances between clinicians and patients' families regarding preferences for how prognostic information is disclosed [7], this topic has been understudied in neurocritical care-somewhat surprisingly, given the attention that developing disease-specific severity scores and avoiding self-fulfilling prophesies have received. Regarding communication of prognostic estimates and uncertainty, Muehlschlegel and colleagues [8] conducted extensive individual interviews of 16 surrogate decision makers for critically ill traumatic brain injury (TBI) patients who had participated in goals-of-care decision-making at two major academic medical centers within 2 years, as well as 20 physicians from seven centers with experience in TBI management. The study reported a discordance between surrogates and physicians with regards to preference for quantitative statements of prognosis, with $82 \%$ of surrogates expressing preference for numeric estimates and $75 \%$ of physicians reporting that they intentionally avoid hard numbers. Furthermore, 57\% of surrogates reported struggling with prognostic uncertainty, and only $19 \%$ felt that uncertainty was an expected part of decision-making.

These findings highlight issues that will be difficult to resolve when it comes to design future interventions to improve goals-of-care decisions in neuro-ICUs. The key concerns that physicians cited for avoiding quantitative estimates-namely, difficulty in applying severity score calculators to individual patients and discomfort with how families may interpret estimated probabilities - are undeniably valid in the context of available research $[9,10]$. Yet, as the study authors point out-in addition to the hunger from surrogates for more concrete prognostic information-the current standards for assessing quality among decision aids designed to assist with shared decision-making highlight the importance of citing numeric probabilities from the literature, in the interest of adhering to an "evidence-based" approach [11]. As there is an evidence that (1) knowledge of probabilities estimated from disease-specific severity scores influences physician judgment [12] and (2) subjective physician judgment of patient prognosis may in fact be more accurate than severity score estimates [9], it may be prudent still for any neuro-ICU decision aids in development to avoid numeric estimates of prognosis, despite what might be concern from experts used for evaluating such tools.

Finally, while developing best practices for families certainly presents challenges, incapacitated patients with severe ANI admitted without any surrogate decision maker are at particularly high risk for care decisions that may not represent what their wishes might have been. Their care already being susceptible to clinicians' biases, these patients in some circumstances may be profoundly affected by legal considerations. Sequeira and Lewis [13] present a case of a homeless patient who was projected to be in a long-term vegetative state following an intraparenchymal hemorrhage and cardiac arrest, with need for long-term dialysis. They report that New York state law requires court approval for all decisions to withdraw life support from unbefriended patients, unless the patient will imminently die regardless of what is done. The patient was 
ultimately transferred to a long-term care facility with a tracheostomy and feeding tube. The article highlights the variability that exists among state laws and professional society guidelines regarding how physicians should proceed in such situations. The authors chose to focus their conclusion on the importance of promoting advanced directives before patients become incapacitated due to medical tragedies. For an equally important attempt to standardize the approach to unbefriended, incapacitated patients, an acceptance of mandatory institutional review for all care-withdrawal cases-as opposed to unilateral decision-making by the clinical team-would seem like a good place to start [14].

\section{Compliance with Ethical Standards}

Conflicts of interest Dr. Hwang has received recent funding from the American Brain Foundation, the Apple Pickers Foundation (Westerly, RI), the Neurocritical Care Society, and the National Institute on Aging (for Loan Repayment). He has also received recent speaking fees from Pennsylvania State University, Mayo Clinic, and the Society of Critical Care Medicine and modest book royalties from Oxford University Press and the Neurocritical Care Society. He has received modest fees for medico-legal consulting.

\section{References}

1. Davidson JE, Jones C, Bienvenu OJ. Family response to critical illness: postintensive care syndrome-family. Crit Care Med. 2012;40(2):618-24.

2. Davidson JE, Aslakson RA, Long AC, et al. Guidelines for family-centered care in the neonatal, pediatric, and adult ICU. Crit Care Med. 2017;45(1):108-28.

3. Shaffer KM, Jacobs JM, Coleman JN, et al. Anxiety and depressive symptoms among two seriously medically ill populations and their family caregivers: a comparison and clinical implications. Neurocrit Care. 2016. doi:10.1007/s12028-0160358-3.

4. Zigmond AS, Snaith RP. The hospital anxiety and depression scale. Acta Psychiatr Scand. 1983;67(6):361-70.

5. Creutzfeldt CJ, Hanna MG, Cheever CS, et al. Palliative care needs assessment in the neuro-ICU: effect on family. Neurocrit Care. 2017. doi:10.1007/s12028-017-0426-3.

6. Carson SS, Cox CE, Wallenstein S, et al. Effect of palliative careled meetings for families of patients with chronic critical illness: a randomized clinical trial. JAMA. 2016;316(1):51-62.

7. White DB, Engelberg RA, Wenrich MD, Lo B, Curtis JR. The language of prognostication in intensive care units. Med Decis Mak. 2010;30(1):76-83.

8. Quinn T, Moskowitz J, Khan MW, et al. What families need and physicians deliver: contrasting communication preferences between surrogate decision-makers and physicians during outcome prognostication in critically ill TBI patients. Neurocrit Care. 2017. doi:10.1007/s12028-017-0427-2.

9. Hwang DY, Dell CA, Sparks MJ, et al. Clinician judgment vs formal scales for predicting intracerebral hemorrhage outcomes. Neurology. 2016;86(2):126-33.

10. Zier LS, Sottile PD, Hong SY, Weissfield LA, White DB. Surrogate decision makers' interpretation of prognostic information: a mixed-methods study. Ann Intern Med. 2012;156(5):360-6.

11. Stacey D, Bennett CL, Barry MJ, et al. Decision aids for people facing health treatment or screening decisions. Cochrane Database Syst Rev. 2017;4:CD001431.

12. Turnbull AE, Krall JR, Ruhl AP, et al. A scenario-based, randomized trial of patient values and functional prognosis on intensivist intent to discuss withdrawing life support. Crit Care Med. 2014;42(6):1455-62.

13. Sequeira AL, Lewis A. Ethical and legal considerations in the management of an unbefriended patient in a vegetative state. Neurocrit Care. 2017. doi:10.1007/s12028-017-0405-8.

14. White DB, Curtis JR, Lo B, Luce JM. Decisions to limit lifesustaining treatment for critically ill patients who lack both decision-making capacity and surrogate decision-makers. Crit Care Med. 2006;34(8):2053-9. 